\title{
ESPAÇO URBANO: UMA ANÁLISE DAS AVENIDAS YANDARA E SENADOR HÉLIO CAMPOS NO MUNICÍPIO DE RORAINÓPOLIS - RR
}

Felipe Melo de Souza ${ }^{1}$

Adriana Roseno Monteiro ${ }^{2}$

Antonio Tolrino de Rezende Veras ${ }^{3}$

\begin{abstract}
RESUMO
Com o intuito de fortalecer as discussões sobre o espaço urbano, este trabalho é fruto de uma pesquisa realizada na cidade de Rorainópolis/RR, trazendo uma contribuição ao estudos das relações com o espaço urbano a partir da análise das avenidas Dra. Yandara e Senador Hélio Campos, com o objetivo de analisar os fixos e fluxos nas avenidas a partir da análise dos aspectos considerados por Del Rio como forma de entender a dinâmica no processo de produção do espaço, estabelecendo suas características, como resultado as características e as diferenças entre a relação ao uso e organização do espaço urbano.
\end{abstract}

PALAVRAS-CHAVE: Análise Urbana. Espaço Urbano. Rorainópolis/RR.

\section{URBAN SPACE: AN ANALYSIS OF THE AVENUES AND SENATOR YANDARA HÉLIO FIELDS IN Rorainópolis COUNTY - RR}

\section{ABSTRACT}

In order to strengthen discussions on urban space, this work is the result of a survey conducted in the city of Rorainópolis / RR, bringing a contribution to studies of the relationship with the urban space from the analysis of the avenues and Senator Dr. Yandara Helium fields, with the aim of analyzing the fixed and flows in the avenues from the analysis of the aspects considered by Del Rio as a way to understand the dynamics of the production process space, establishing its characteristics as a result

\footnotetext{
${ }_{1}^{1}$ Mestrando em Geografia do PPG - GEO - UFRR ; felipesouza@dau.ufrr.br

${ }^{2}$ Mestranda do PPG-GEO/UFRR, drycaluz@yahoo.com.br

3 Doutor do Instituto de Geociências/UFRR, tolrino@usp.br
} 
Revista Nacional de

Gerenciamento de Cidades

of the characteristics and differences between the relationship the use and organization of urban space.

KEYWORDS: Urban Analysis. Urban Space. Rorainópolis / RR.

\section{ESPACIO URBANO: UN ANÁLISIS DE LA AVENIDAS Y SENADOR YANDARA HÉLIO CAMPOS EN RORAINÓPOLIS CONDADO - RR}

\section{RESUMEN}

Con el fin de fortalecer el debate sobre el espacio urbano, este trabajo es el resultado de una encuesta realizada en la ciudad de Boa Vista / RR, trayendo una contribución al estudio de la relación con el espacio urbano a partir del análisis del Dr. avenidas. Yandara y el Senador Hélio campos, con el objetivo de analizar las y los flujos fijos en las avenidas del análisis de los aspectos considerados por Del Río, como una manera de entender la dinámica en el proceso de producción del espacio, el establecimiento de sus características, como consecuencia de las características y diferencias entre la relación el uso y la organización del espacio urbano.

PALABRAS CLAVE: Análisis Urbano. Espacio Urbano. Rorainópolis / RR.

\section{INTRODUÇÃO}

O objeto de estudo são as avenidas Dra. Yandara e Senador Hélio Campos, localizada no bairro Centro do município de Rorainópolis, no qual concentra grande e importante área comercial da cidade, a proposta da escolha da área localizada no bairro do centro, bem como o recorte é em virtude da dinâmica e do valor histórico das avenidas, é em função da localização, situadas no núcleo gênese da cidade.

Com uma dinâmica bem diferente entre as áreas das duas avenidas. A área está localizada num ponto importante para a cidade, principal ponto de entrada da cidade, tem sua importância por possuir a maior área comercial do município distribuída entre comércios e serviços para a população, dentre eles terminal das vans de transporte, oficina de moto, restaurante, bar, borracharia, entre outros serviços.

Com relação aos referenciais teóricos, temos Santos, utilizando seus conceitos de Rugosidades e espaços luminosos e opaco, como forma de 
Revista Nacional de

Gerenciamento de Cidades

compreender a dinâmica urbana das avenidas, considerando seus elementos urbanos e arquitetônicos presente e através das considerações da análise do espaço urbano, como referência Del Rio, 1990 para sistematizar nossa análise, como complemento.

O objetivo deste trabalho é analisar os fixos e fluxos nas avenidas Dra. Yandara e Senador Hélio Campos a partir da análise dos aspectos considerados por Del Rio como forma de entender a dinâmica das referidas avenidas no processo de produção do espaço, estabelecendo suas características, de forma a compreender e de ser uma diretriz para os futuros trabalhos a serem realizados nos municípios, corroborando para os possíveis análises em outros municípios do estado.

A metodologia do trabalho consistiu em ações de pesquisa qualitativas, combinando informações conceituais e descritivas para a compreensão do objeto estudado. Para isso utilizou-se a pesquisa bibliográfica, para coleta de dados, com o intuito de propor uma pesquisa de método dedutivo. No segundo momento temos a pesquisa de campo, que contou com uma análise visual nas avenidas Dra. Yandara e Senador Hélio Campos como forma de compreender as características responsáveis pela dinâmica urbana.

\section{FIXOS E FLUXOS: UM OLHAR SOBRE AS AVENIDAS DRA. YANDARA E SENADOR HÉLIO CAMPOS E SUA DINÂMICA ESPACIAL}

A paisagem do município de Rorainópolis nos traz uma série de características ligadas a produção do espaço, dentre elas e um dos elementos a rua, apresenta uma dinâmica que favorece a relação da cidade com seus habitantes, criando um laço de afetividade através de sua interação do homem com espaço, produzindo elementos que podem ser analisados como forma de criar subsídios para análise de sua produção do espaço, com destaque:

A paisagem é o conjunto das coisas que se dão diretamente aos nossos sentidos, a configuração territorial é o conjunto total, integral, de todas as coisas que formam a natureza em seu aspecto superficial e visível, e o espaço é o resultado de um matrimônio ou de um encontro sagrado 
Revista Nacional de

Gerenciamento de Cidades

nesse processo histórico das relações de valor com o espaço urbano, como podemos observar na área central da cidade de Rorainópolis. Contribui para essa reflexão Carlos:

Portanto, deixando de lado postulados e afirmações dogmáticas, podemos tomar como ponto de partida para o desenvolvimento do raciocínio capaz de produzir uma "leitura geográfica sobre a cidade" a ideia de cidade como construção humana, produto histórico-social, contexto no qual a cidade aparece como trabalho materializado, acumulado ao longo de uma série de gerações, a partir da relação da sociedade com a natureza. (2007, p. 20)

\section{A INFLUÊNCIA DOS FIXOS PELOS FLUXOS NA DINÂMICA DO ESPAÇO URBANO NA AVENIDA DRA YANDARA}

$\mathrm{Na}$ avenida Dra. Yandara observa-se que tem uma dinâmica completamente diferente da avenida senador Hélio Campos, um primeiro olhar sobre a avenida percebe-se claramente um movimento intenso gerado de seus fixos, que acabam provocando um grande fluxo ao longo de todo o dia, criando uma relação direta entre seus atores e a avenida.

Com relação a avenida aqui destacamos através do elemento rua propriamente dita, um fluxo provocado em função da relação que se criou com os fixos, nesse caso destacamos dois fixos ligados ao transporte, ou seja gerando um impacto de circulação de pessoas, estabelecendo uma rede de circulação dos fluxos:

1. Ponto das van: Representados pelo ponto das Vans, que leva e traz as pessoas provenientes das vicinais do município;

2. Ponto de moto táxi: Ponto de moto táxi que é responsável por transportar as pessoas vinda de outras localidades e leva-las até o seu destino final dentro da cidade de Rorainópolis;

3. Ponto dos motoristas de caminhões de apoio dos agricultores: responsáveis por leva e trazer os agricultores com sua produção das vicinais até o município de Rorainópolis; 

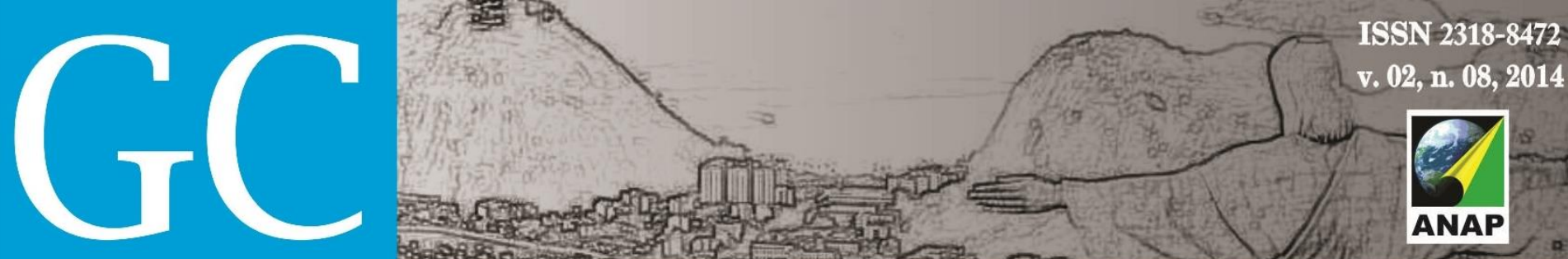

Revista Nacional de

Gerenciamento de Cidades

Temos uma preocupação dos proprietários em valorizar suas fachadas, sendo que também é na referida avenida que se encontram os principais pontos de apoio da cidade, que é o supermercado Figueiredo que está localizado na frente dos pontos das vans e que acaba sendo utilizado como terminal das vans de transporte entre a capital Boa Vista e as Vicinais.

De uma forma geral a avenida Dra. Yandara tem as fachadas das edificações muito mais valorizadas, uma vez que seu eixo é o principal de ligação com os demais bairros do município de Rorainópolis. $O$ interessante é que quase em sua totalidade os serviços são privados, com exceção do mercado municipal que é público, ou seja, uma avenida que não foi planejada ou organizada de forma setorizada, criando uma ocupação urbana de forma espontânea.

Figura 02: Lojas de Eletroeletrônicos e Móveis na av. Dra. Yandara

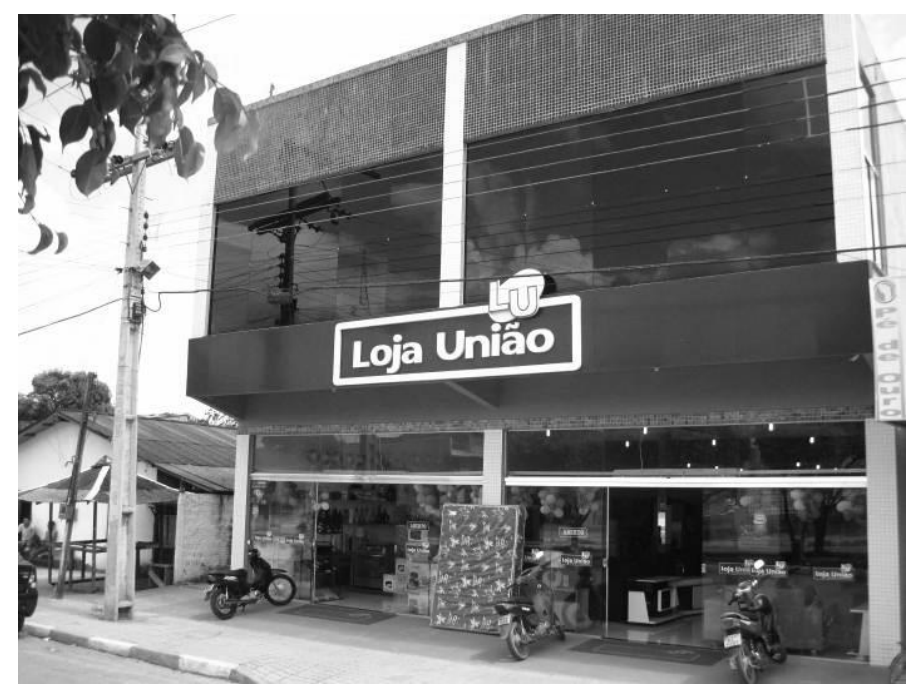

Um dos destaque também é com relação ao transporte, onde os serviços de moto táxi também são significativos para o deslocamento dos passageiros dentro da cidade. Nota-se que o transporte particular na cidade de Rorainópolis é mais expressivo e que a cidade ainda não possui transporte público, que de acordo com Souza, é uma realidade evidente no Brasil. 


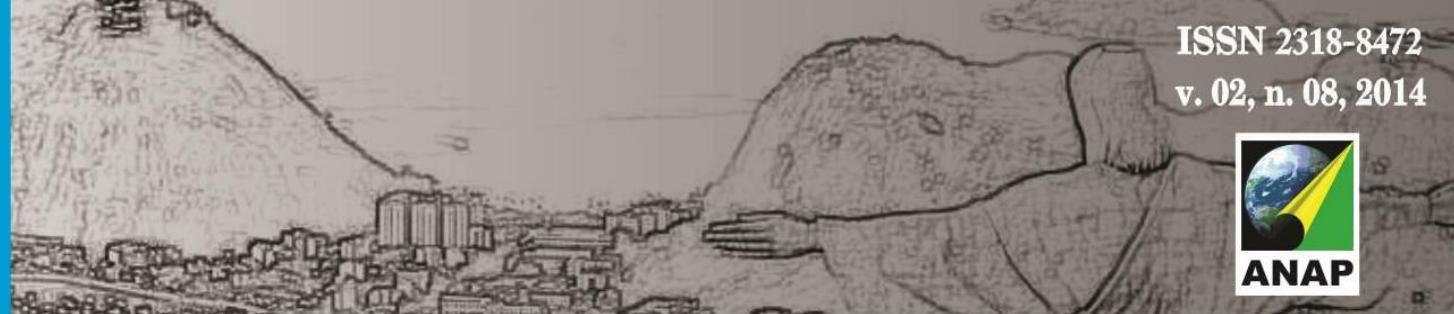

Revista Nacional de

Gerenciamento de Cidades

A prioridade do veículo particular de transporte de passageiros é evidente no Brasil; ela reflete tanto distorções de mentalidade e defeitos de planejamento quanto, sem dúvida, a influência da poderosíssima indústria automobilística e de seus interesses. (SOUZA, 2003, p. 85)

De acordo com Meneses, 2004, p.752 "é tempo de desarticular todas as categorias geo-culturais que pretendem preservar o seu estatuto hegemônico e organizar o espaço e o tempo em termos epistemológicos, fazendo do saber um processo perpétuo e um alicerce central da proposta de uma sociedade mais justa e válida.

Mas outra característica relevante desta avenida é a presença de grandes comércios atacadista, o que a valoriza ainda mais.

\section{ENTRE AS RUGOSIDADES E OPACIDADES DA AV. SENADOR HÉLIO CAMPOS}

A avenida senador Hélio Campos tem características que diferem da avenida Dra. Yandara, essa diferença é facilmente observada num primeiro olhar sobre a referida avenida, pois temos uma relação com o espaço urbano muito diferente em função principalmente dos fixos e fluxos, que na referida análise ocorre por conta da relação entre os fixos e fluxos, na reflexão de Santos, 2006 p. 92.

\footnotetext{
Chamemos rugosidade ao que fica do passado como forma, espaço construído, paisagem, o que resta do processo de supressão, acumulação, superposição, com que as coisas se substituem e acumulam em todos os lugares. As rugosidades se apresentam como formas isoladas ou como arranjos. É dessa forma que elas são uma parte desse espaço fator. Ainda que sem tradução imediata, as rugosidades nos trazem os restos de divisões do trabalho já passadas (todas as escalas da divisão do trabalho), os restos dos tipos de capital utilizados e suas combinações técnicas e sociais com o trabalho.
}

Com base na reflexão de Santos observamos que a avenida senador Hélio Campos apresenta uma rugosidade em função da cristalização do tempo refletida na relação dos habitantes com o espaço urbano, possuindo uma série de imóveis abandonados, incluindo hotéis e serviços, representando uma constituição de que a 

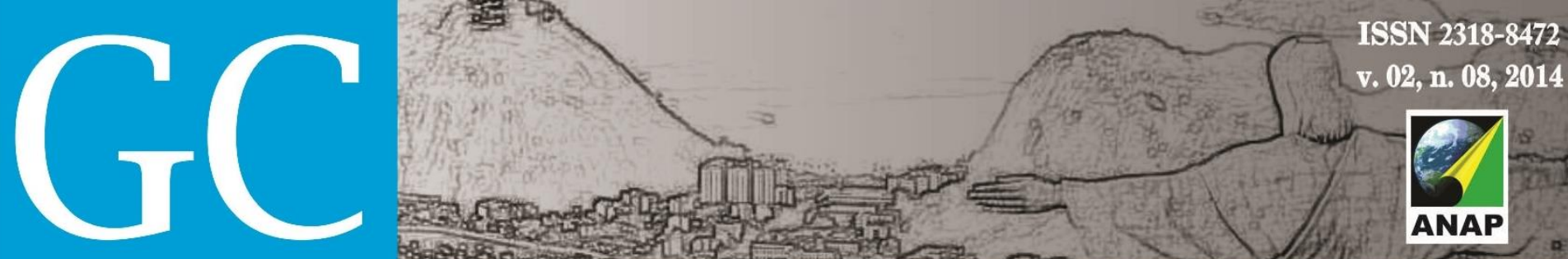

Revista Nacional de

Gerenciamento de Cidades

produção do espaço e seu reflexo na dinâmica do espaço urbano é fruto das relações entre fluxos e fixos.

Uma outra característica é apresentada por Santos e Silveira (2001, p. 264) que afirmam que: chamaremos de espaços luminosos aqueles que mais acumulam densidades técnicas e informacionais, ficando assim mais aptos a atrair atividades com maior conteúdo em capital, tecnologia e organização. Por oposição, os subespaços onde tais características estão ausentes seriam os espaços opacos.

Figura 03: Avenida Dra Yandara com as edificações presentes de sua gênese com poucas modificações

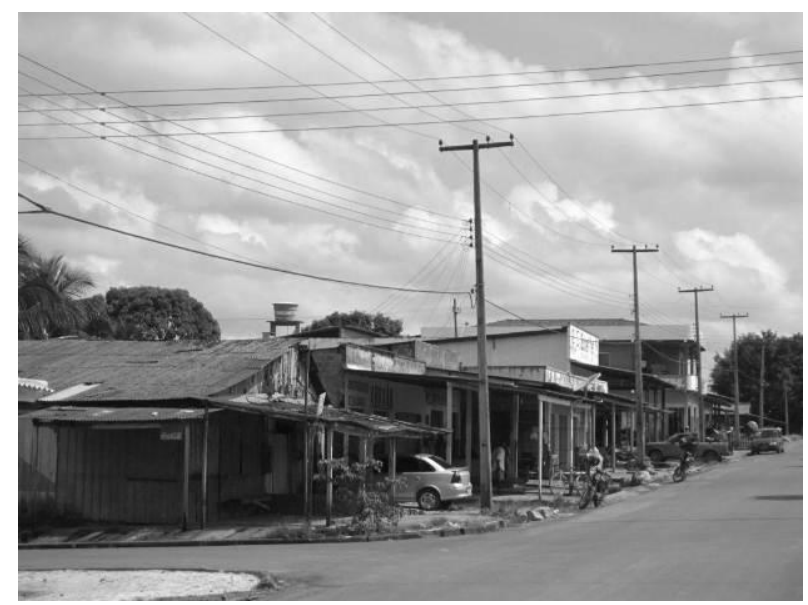

Fonte: SOUZA, Felipe Melo, 2012

Ao circularmos pela avenida Sen. Hélio Campos, temos um grande contraste com a Av. Dra. Yandara em vários aspectos: o $1^{\circ}$ deles é o fato de grande parte das edificações possuírem em suas fachadas uma cobertura, como pode ser observado na figura 03, traz um aspecto remetendo a forma de construção ainda da predominância inicial das edificações, o que nos faz identificar além da opacidade apontada por Santos uma rugosidade, através dos elementos encontrados nas características de sua estrutura atual. 


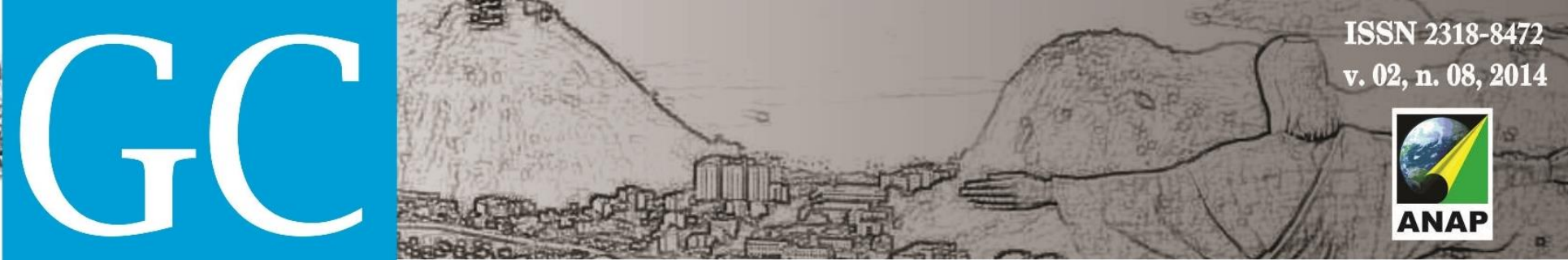

Revista Nacional de

Gerenciamento de Cidades

Um dos pontos em destaque é a das coberturas das edificações da avenida senador Hélio Campos que pela grande incidência solar durante a manhã, possui em grande parte das fachadas das edificações uma varanda com cobertura, alterando a configuração das edificações em função da sua necessidade em virtude dos elementos de conforto ambiental.

A avenida senador Hélio Campos, paralela a BR-174 está desconectada do núcleo principal da cidade, sendo a avenida Dra. Yandara, principal ligação com os demais bairros de Rorainópolis/RR, sendo talvez esse fato um dos elementos de opacidade da avenida senador Hélio Campos, além da falta de infraestrutura.

Uma característica das edificações é que permanecem com as mesmas características iniciais de sua construção com ausência de uma unidade e sem os recuos necessários que deveriam está previsto no código de obras do município, a imagem 07 ilustra bem, outro, detalhe é também com relação à rede de distribuição de energia elétrica que posicionou os postes de energia no meio da calçada de circulação das pessoas, prejudicando sua circulação.

Com uma perspectiva positiva, temos apesar dos problemas enfrentados relacionados à própria valorização da avenida, tendo em vista a saturação da avenida Dra.Yandara, a av. Senador Hélio Campos tem um potencial para valorização, pois possui muitos imóveis fechados e estado de abandono.

Uma outra possível intervenção é a implantação de fixos ao longo da avenida, podendo aqui ser representados com a instalação de setores ligados ao governo municipal, além de uma proposta de recuo para as edificações obedecendo a um gabarito, construção de passeio público para a circulação dos pedestres. Com isso a proposta é torna o lugar atrativo, através dos fluxos e ainda ser com o passar do tempo um espaço luminoso, principalmente para fomento de futuros investimentos, pois sua localização reforça a importância da avenida como elemento estratégico de desenvolvimento.

PROCESSO, ESTRUTURA, FUNÇÃO E FORMA. 


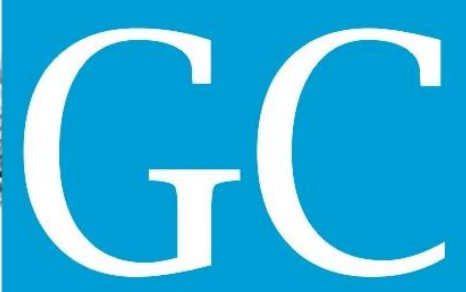

Revista Nacional de

Gerenciamento de Cidades

São a partir desses entendimentos que se podem ser definidas as categorias filosóficas - forma, função, estrutura e processo como método de análise da Geografia. Para Milton Santos (1985, p. 50)

[...] a forma é o aspecto visível de uma determinada coisa. São os objetos e arranjos de objetos que compõe o espaço, isto é, casas, edifícios de condomínios, parques, escolas avenidas e etc., tudo gerado historicamente, organizando o presente e projetando o futuro. A função é a atividade desempenhada pela forma. Ela dá sentido á forma visto que um objeto no espaço não subsiste desprovido de tarefa e, por outro lado, a tarefa não pode ser desempenhada sem a forma, daí a relação direta entre as duas. Um terceiro aspecto da análise é a estrutura. Santos assinala que "estrutura implica a inter-relação de todas as partes de um todo; o modo de organização ou construçãoll (1985,p.50).

É o aspecto "invisívelll construído pela inter-relação das diversas funções desempenhadas pelas/nas formas. Por isso para compreendê-la é preciso sempre considerar a dinâmica social de cada período. Por fim, o processo é definido como uma ação contínua, desenvolvendo-se em direção a um resultado qualquer, implicando conceitos de tempo (continuidade) e mudança

\section{ANÁLISE DO DESENHO URBANO A PARTIR DE DEL RIO}

Com base nos elementos apontados por Del Rio, uma análise urbana na cidade, utilizou-se dos elementos circulação viária estacionamento e espaços livres e mobiliário urbano, devido as características da área analisada como forma também de contribuir para o estudo sobre a dinâmica urbana no município, considerando os abaixo:

\section{CIRCULAÇÃO VIÁRIA E ESTACIONAMENTO:}

A circulação viária é um dos elementos mais poderosos para a estruturação da imagem urbana (LYNCH1960, APPLEYARD 1981, SHIRVAN I 1985. 26) e, portanto, não pode ser tratada apenas como um sistema de movimento; um dos fatores básicos na democratização da cidade uma vez definidora da acessibilidade (LYNCH 1981); a circulação viária, o transporte público e o estacionamento devem ser entendidos como vitais para a animação e a sobrevivência social e econômica de uma área, em soluções conciliadoras. (DEL RIO, 1990) 
Revista Nacional de

Gerenciamento de Cidades

Equador e Novo Paraíso, São João da Baliza, Caroebe, São Luiz do Anauá, o que fortalece ainda mais seu potencial para o comércio da região, um outro fato é relacionado a própria dinâmica da produção do espaço, que está em constante transformação, sendo visível para quem passa pelo Município, edifícios sendo reformados, construções de novas edificações, a cidade em constante transformação.

Outra consideração a fazer é relacionado a sua dinâmica, a própria avenida Dra. Yandara, na área do centro da cidade já está saturada sem espaço para uma futura expansão das edificações em função das mesmas estarem geminadas umas nas outras. Por outro lado temos a Av.Senador Hélio Campos que possui um potencial enorme para uma melhoria na qualidade das edificações e uma futura expansão de seu comércio.

Um dos fatores essenciais e urgente que o município deve criar é um plano de ordenamento urbano, através do código de edificações, como forma de organizar e orientar a expansão urbana da cidade, e principalmente a construção e aprovação do plano diretor, que é o principal instrumento para a organização urbana sendo imprescindível para o município de Rorainópolis.

Observamos que o espaço urbano das avenidas em estudo, serviram para ampliarmos o olhar a respeito dos condicionantes responsáveis pelo aumento do fluxo a partir do fixo, que pode ser facilmente visualizado a partir de demais pontos da cidade de Rorainópolis, que possui outras áreas em expansão comercial, e que pode ser compreendida com as considerações de Milton Santos, agregados com as considerações de Del Rio, como forma de fortalecer o entendimento do espaço urbano.

\section{REFERÊNCIAS}

CARLOS, Ana Fani Alessandri. Espaço Urbano: Novos Escritos Sobre a Cidade. FLECH, São Paulo, 2007.

CARLOS, Ana Fani Alessandri; Souza, Marcelo Lopes de; Sposito, Maria Encarnação Beltrão (org). A produção do Espaço Urbano: agentes e processos, escalas e desafios. São Paulo: Contexto, 2011. 
